\title{
Mapping Sensitive Vegetation Communities in Mining Eco- space using UAV-LiDAR
}

This paper was downloaded from TechRxiv (https://www.techrxiv.org).

\section{LICENSE}

CC BY-NC-SA 4.0

SUBMISSION DATE / POSTED DATE

01-11-2021 / 09-11-2021

\section{CITATION}

Banerjee, Bikram; Raval, Simit (2021): Mapping Sensitive Vegetation Communities in Mining Eco-space using UAV-LiDAR. TechRxiv. Preprint. https://doi.org/10.36227/techrxiv.16912138.v1

DOI

10.36227/techrxiv.16912138.v1 


\title{
Mapping Sensitive Vegetation Communities in Mining Eco-space using UAV-LiDAR
}

\author{
Bikram Pratap Banerjee ${ }^{1,2}$, and Simit Raval ${ }^{2, *}$ \\ ${ }^{1}$ Agriculture Victoria, Grains Innovation Park, 110 Natimuk Road, Horsham, VIC 3400, Australia; \\ bikram.banerjee@agriculture.vic.gov.au \\ ${ }^{2}$ School of Minerals and Energy Resources Engineering, University of New South Wales, Sydney, Australia NSW \\ 2052; simit@unsw.edu.au
}

* Corresponding Author. Tel.: +61 (2) 93855005

\begin{abstract}
Near earth sensing from unmanned aerial vehicles or UAVs has emerged as a potential approach for fine-scale environmental monitoring. These systems provide a cost-effective and repeatable means to acquire remotely sensed images in unprecedented spatial detail and high signal-to-noise ratio. It is becoming increasingly possible to obtain both physiochemical and structural insights of the environment using state-of-art light detection and ranging (LiDAR) sensors integrated onto UAVs. Monitoring of sensitive environments, such as swamp vegetation in longwall mining areas is important, yet challenging due to their inherent complexities. Current practices for monitoring these remote and difficult environments are primarily ground-based. This is partly due to an absent framework and challenges of using UAV-based sensor systems in monitoring such sensitive environments. This research addresses the related challenges in the development of a LiDAR system including a workflow for mapping and potentially monitoring highly heterogeneous and complex environments. This involves the amalgamation of several design components, which include hardware integration, calibration of sensors, mission planning, and designing of a processing chain to generate usable datasets. It also includes the creation of new methodologies and processing routines to establish a pipeline for efficient data retrieval and generation of usable products. The designed systems and methods were applied on a peat swamp environment to obtain accurate geospatialised LiDAR point cloud. Performance of the LiDAR data was tested against ground-based measurements on various aspects including visual assessment for generation LiDAR metrices maps, canopy height model, and fine-scale mapping.
\end{abstract}

Keywords: Upland swamps, mine surveying, monitoring, environment sustainability, drones and laser scanning.

\section{Introduction}

Extraction and use of minerals is a critical component in the development of current and future societies. In contrast to its benefits there have been concerns about the negative impacts of mining on the economy, society and the environment (Chang et al., 2018). Mining is a temporary use of the land, but its socio-environmental impacts could be long term. Continuous monitoring of the mine environment provides an opportunity for early remediation leading to the long-term sustainability of the environment and mining operations.

\subsection{Coal Mining under upland peat swamp environments}

Mining under economically significant and ecologically sensitive environments such as upland peat swamps pose a unique challenge to the mining industry and government regulators. Upland peat swamps in the Sydney basin bioregion, New South Wales, Australia mostly occur on coastal highland or upland plains of Triassic Sandstone formation (Department of Sustainability, Environment, Water, Population and Communities, 2018) and are technically termed temperate highland peat swamps on sandstone (THPSS). THPSS consists of uniquely diverse ecosystems comprising treeless heaths and sedgelands. These environments play an essential role in filtering and slowly releasing water to downstream watercourses. Additionally, providing habitats for a wide range of animals, including birds, reptiles and frogs. A few of the threatened species with habitat requirements specific to THPSS conditions include the Giant burrowing frog (Heleioporus australiacus), Blue Mountains water skink (Eulamprus leuraensis) and Giant dragonfly (Petalura gigantean) (CoA, 2014). THPSS environments are listed as an endangered ecological community because of their limited distribution and vulnerability to ongoing threats such as underground longwall mining and agriculture (CoA, 2014). These ecological communities may be influenced by underground longwall coal mining 
activities, which have the potential to disrupt the local geology, topography, water regimes and water quality of the THPSS (NSWDP, 2008; CoA, 2014; Vervoort, 2021). Potential impacts include the disruption of water availability and quality, leading to the degradation of the host ecosystem.

Accidental discharge of mine wastewater into drainage lines uphill from the swamps is also recognized as a potential risk (CoA, 2014). Improperly treated mine water discharges include saline discharges (Opitz and Timms, 2016; Greene et al., 2016) and acid mine drainage (Akcil and Koldas, 2006), all of which can degrade freshwater resources and potentially impact sensitive environments and ecosystems (Younger and Wolkersdorfer, 2004). Poor water quality may involve one or more of the following parameters: salinity, turbidity, acidity, metals, organics, and other contaminants of concern, such as toxic algae or radiological elements. However, it is difficult to be definitive about water quality and identify potential short and long-term impacts on swamps with limited monitoring.

\subsection{Existing monitoring technologies}

Several traditional methods are used for monitoring the potential impact of mining on peat swamps. Methods suitable for early identification of impacts include field based geotechnical methods (borehole testing and joint monitoring), geophysical methods (downhole logging, electromagnetic conductivity and ground penetrating radar) and hydrological methods (shallow groundwater monitoring, deep groundwater level or pressure monitoring) (CoA, 2014). However, peat swamps are often located in steep and elevated terrain, and many of these field-based methods are constrained by difficult site access and coverage area, particularly if heavy equipment is necessary. The status of swamps can also be assessed using baseline ecological conditions, which includes vegetation survey (flora census, vegetation community patterns and vegetation condition), fauna (wetland frog, reptile, bird and invertebrate) monitoring, and invasive species monitoring methods (CoA, 2014). These ecological field surveys are limited to monitoring specific locations and rely on data extrapolation. These methods are also subjective to the approach being used to infer the ecological baselines, and usually a set of different ecological surveying methods are needed to establish the condition of swamps.

Remote sensing based methods are alternative approaches to monitor the ecological response of swamp vegetation. These methods involve both passive (multispectral, hyperspectral and thermal) and active (light detection and ranging, i.e. LiDAR and radar) sensing approaches from airborne and satellite systems. Multispectral indices, such as the normalized difference vegetation index (NDVI), indicate vegetation vigour, or greenness, on the assumption that high chlorophyll absorption in plants conveys information on plant health (Gandaseca et al., 2009; Setiawan et al., 2017). The enhanced vegetation index (EVI) has advantages over the NDVI to more accurately interpret vegetation coverage by incorporating corrections for atmospheric and soil influences (Weier and Herring, 2000). Time series EVI data is available in high frequency and has been used for monitoring dynamics of peat swamps (Setiawan et al., 2016). Changes in vegetation distribution within peat swamps can be monitored using high-resolution remote sensing (Jenkins and Frazier, 2010; Zhang et al., 2018). Light detection and ranging (LiDAR) is an active-sensing system which can differentiate between bare earth and 'non-ground' points such as vegetation to monitor biomass (Englhart et al., 2013). Insufficient spatial resolution of aerial and satellite based methods also restricts fine level assessment of vegetation condition in THPSS, and consequently existing studies (Jenkins and Frazier, 2010) have been limited to delineation of swamp boundaries and vegetation baseline estimations.

\subsection{Environmental monitoring using UAVs}

Plant species physiologically adapted to survive in periodically inundated conditions have a competitive advantage in wetlands and swamps. Any alteration in swamp hydrology that causes drying of the peat will reduce this competitive advantage of wetland species and allow species assemblages to shift towards more terrestrial vegetation types (CoA, 2014). Increases in the proportion of terrestrial species in a swamp may indicate changing swamp hydrology. No change in the proportion of terrestrial species (or change within equilibrium limits) indicates the stability of hydrology and peat moisture levels. This baseline composition of species within the equilibrium limit is regarded as the characteristic vegetation composition, which is unique for each environment, including THPSS. Identification of individual vegetation species or assemblages in THPSS is critical to characterize their vegetation composition, and is the first step towards monitoring the changing health of the micro-ecosystem under natural or anthropogenic stresses (Banerjee et al., 2017; Banerjee et al., 2020). 


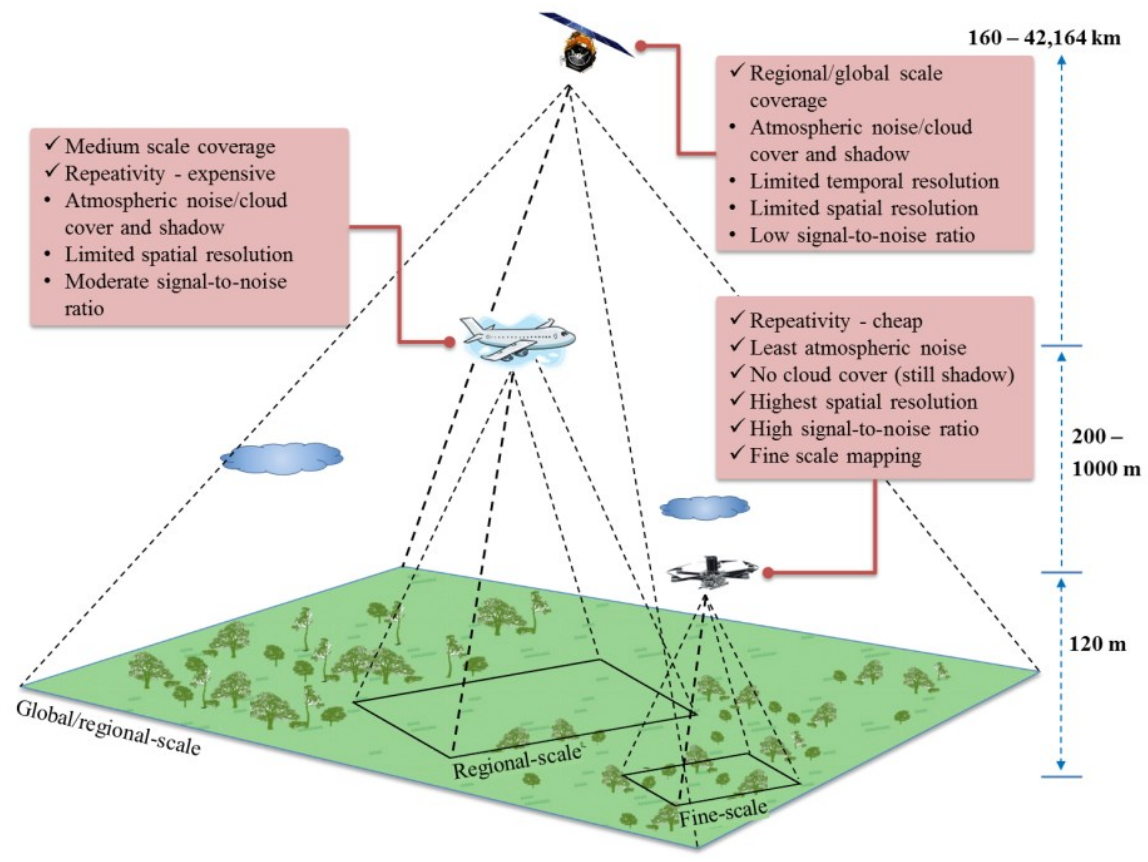

Fig. 1 A comparison of satellite, airborne and unmanned aerial vehicle (UAV) remote sensing systems for environmental monitoring applications.

\subsection{UAV-LiDAR scanning in environmental monitoring}

Recent developments in the miniaturization of aerial robotic platforms and electro-optical sensors have established a new era of aerial remote sensing using unmanned aerial vehicles (UAVs). The unprecedented resolution, high signal-to-noise ratio, operational flexibility, ability to access remote locations, ease of use and low-cost have attracted interest from both the scientific and commercial communities (Colomina and Molina, 2014; Ren et al., 2019). Such features make them suitable for fine-scale environmental monitoring compared to satellite or airborne systems (Fig. 1). UAVs with optical and infrared cameras have been used in THPSS, however to date the approach has been limited to the detection of a single species (Gleichenia dicarpa) (Strecha et al., 2012) and the mapping of vegetation community boundaries (Lechner et al., 2012). Studies were undertaken to test the potential of a UAV-hyperspectral system to map five swamp species (Allocasuarina littoralis, Empodisma minus, Lepidosperma limicola, Lepidosperma neesii and Pteridium aquilinum) in THPSS environments (Banerjee et al., 2020; Banerjee and Raval, 2021). These studies demonstrate the advantage of both high spatial and spectral resolutions for effectively assessing vegetation in spectrally complex swamp environments.

UAV-LiDAR system is a cutting-edge technology that is and finding increased use in different applications. Jaakkola et al. (2010) developed and demonstrated the potential of a UAV-LiDAR system in a forestry application. Integration of lightweight LiDAR sensors with rotary type UAVs provided the benefit of using them more effectively in restricted environments to obtain high-resolution 3D datasets at unprecedented detail. The high density of the LiDAR datasets directly translates to the accuracy of the derived secondary products such as topographic (Lin et al., 2011) and vegetation (Wallace et al., 2014) metrices. Banerjee et al. (2018) used optical imaging data and structural metrices from UAV-LiDAR for mapping complex vegetation communities in upland peat swamps. Therefore, accurate estimation of these structural metrices is crucial to environmental applications such as generation of topographic and canopy models, identification of vegetation types and attributes such as leaf area index. Finally, UAV-LiDAR systems are accurate, easy to use and have lower operational costs than traditional airborne laser scanning surveys, making them suitable for recurrent use in environmentally sensitive areas for condition assessment and reporting.

To this end, this study focuses on using a UAV-LiDAR system in mapping sensitive vegetation communities in a coal mining area. The work involved detailed protcols of (1) collecting UAV-LiDAR data, including procedures for filtering and generating a coherent point cloud, (2) processing to generate structural metrices, (3) generating the canopy height model, and (4) classification of sensitive vegetation communities using different algorithms. 


\section{Materials and Methods}

\subsection{Study area and identification of vegetation of interest}

Temperate highland swamps on sandstone (THPSS) are critically endangered ecosystems (CoA, 2014) distributed in the Blue Mountains, Lithgow, Southern Highlands and Bombala regions in New South Wales, Australia. This study focused on two swamps in a THPSS site in the Southern Highlands, located near Wollongong, southwest of the city of Sydney, Australia.

The two swamps have a complex distribution of several species and vegetation communities, which exist as shrub-type vegetation thickets (Banksia and Tea-tree), and Sedgeland-Heath complexes (Cyperoid, Restioid and Sedgelands) (NPWS, 2003; Jenkins and Frazier, 2010). Selection of species for a vegetation monitoring and classification study, needs to consider the ability to both map the vegetation components (or classes) and their ecological significance. A total of eight vegetation classes were selected with five swamp vegetation classes and three non-swamp vegetation classes. A set of five swamp vegetation classes were identified based on their abundance and sensitivity to anthropogenic impacts: Dagger hakea (Hakea teretifolia), Grass tree (Xanthorrhoea resinosa), Pouched coral fern (Gleichenia dicarpa), Heath-leaved banksia (Banksia ericifolia), and Sedgeland complex (Empodisma minus, Gymnoschoenus sphaerocephalus, Lepidosperma limicola, Lepidosperma neesii, Leptocarpus tenax and Schoenus brevifolius). Presence of certain non-swamp or terrestrial vegetation species can indicate potential alteration of swamp hydrology. Therefore, a set of three non-swamp vegetation classes were also identified: Black sheoak (Allocasuarina littoralis), Bracken fern (Pteridium aquilinum), and Eucalyptus trees. The total list of classes and corresponding species was identified through several field campaigns and consultation with expert advice from field ecologists.
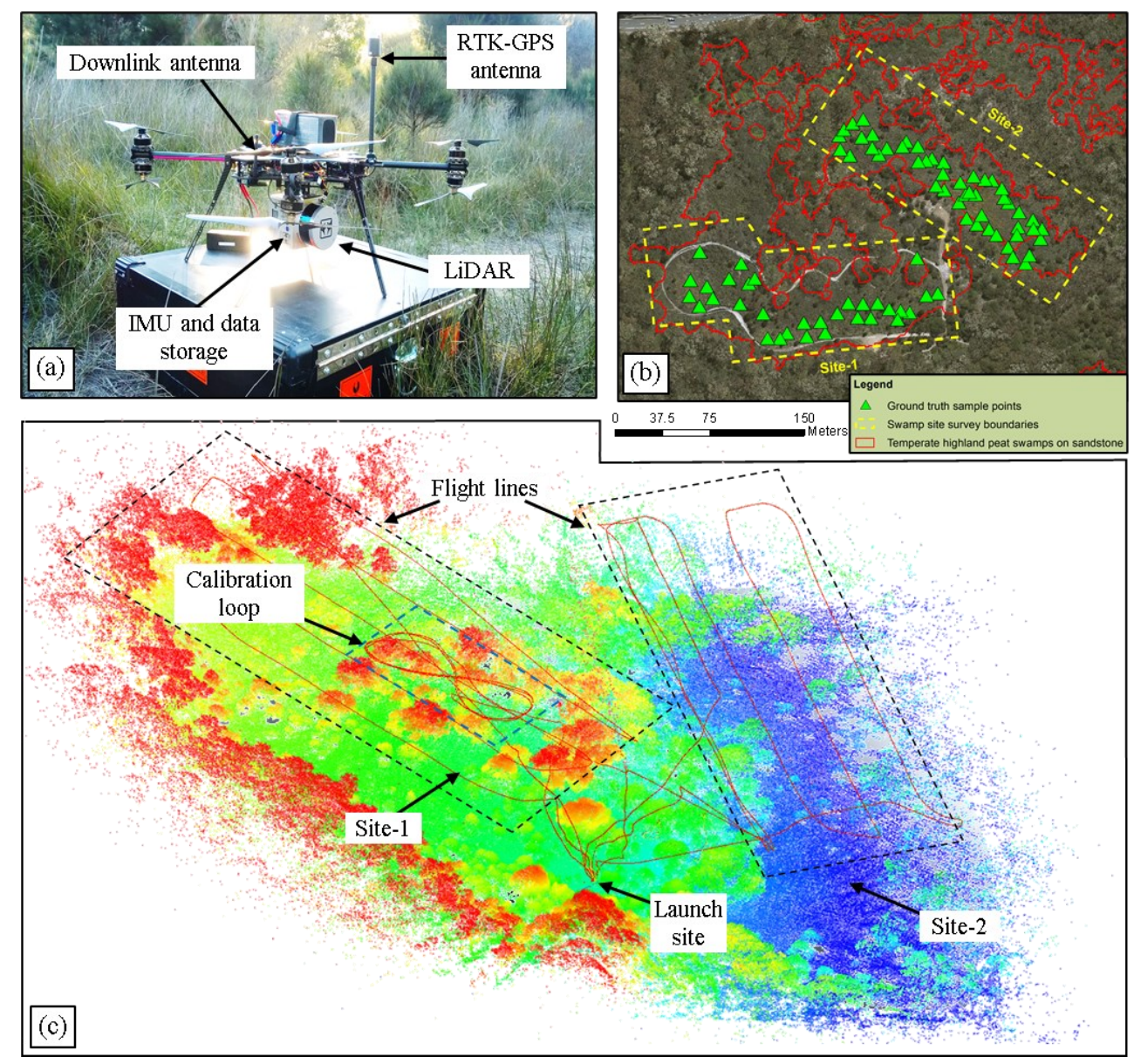

Fig. 2 (a) The integrated UAV-LiDAR system used in the study environment, (b) map view of the study area with detailed view of the swamp site survey boundaries (site-1 and site-2), ground truth sample locations (green triangles) and THPSS boundaries, and (c) 3D subsampled point cloud view with the calibration loop and flight lines for data acquisition. 


\subsection{Sampling design and field measurement}

Ground truth data includes a set of labels on the images intended for defining a model for classification or parameter retrieval. A proper ground truth sampling strategy is essential to eliminate significant biases from the leaking into the process (Congalton, 1991). Furthermore, to ensure that the ground data is representative of the spatial population, a suitable sample design must be chosen. Stratified random sampling is a method of selecting in which the elements of the population are allocated into sub-populations (e.g. strata) before the sample is taken, and then each stratum is randomly sampled (Brogaard and Ólafsdóttir, 1997). This sampling approach is used when specific information about certain subpopulations and increasing precision of the estimates for the entire population is desired (Cochran, 1977; Clark and Hosking, 1986). In this study, a similar stratified sampling approach has been used. A total of 80 locations for ground truth sample collection were identified using this sampling approach within the shrub-type swamp vegetation classes only (i.e. Grass tree, Pouched coral fern and Sedgeland complex) for the field survey, i.e. a total of 32 locations for swamp site-1 and 48 locations for swamp site-2 (shown as green triangles in Fig. 2 (b)). Coordinates of each location were measured using the ground-based realtime kinetic - differential global positioning system RTK-DGPS unit (Leica Viva GS15 GPS system) within $3 \mathrm{~mm}$ of absolute accuracy. A set of four discrete ground truth points were identified around each location at $1 \mathrm{~m}$ distances in the North, East, South and West directions using a compass as reference. This produced a total of $80 \times 4=320$ ground truth points for the shrub-type swamp vegetation. For each ground truth point, the species composition and canopy height of the vegetation were recorded.

Canopy Height Measurement - The canopy height was measured using the vertical graduated scale with a pointed bottom end. Due to the varying degree of density, in areas of high compaction of the shrub-type swamp vegetation it was difficult to ensure that the bottom end of the graduated scale reached the true soil surface. Therefore, several sets of measurements were taken within $(50 \mathrm{~cm})$ the vicinity of the sampling point to correctly identify the correct canopy height. In areas of highly fragile canopies several measurements ( 5 to 10) were collected within $(50 \mathrm{~cm})$ the vicinity of the point and the median value was used as the representative measurement.

\subsection{UAV-LiDAR Scanning}

This section describes the method of developing of a UAV-LiDAR system and workflow including description of the LiDAR sensor used, procedures for system integration and aerial data acquisition, conversion of raw data to point cloud, and preprocessing of the generated point cloud.

\subsubsection{LiDAR sensor}

In this study, a mobile integrated LiDAR system (Phoenix Aerial Scout) was used. The primary sensor on-board the UAV is a Velodyne PUCK LiDAR scanning system. The internal laser sensor has a maximum range of $120 \mathrm{~m}$ and a range of $80 \mathrm{~m}$ at $60 \%$ target reflectivity, which produces a typical range accuracy of $\pm 3 \mathrm{~cm}$ with a range resolution of $2 \mathrm{~mm}$. The laser sensor records ranges and intensities for up to two echoes per pulse. It has a rectangular aperture beam width of $9.5 \mathrm{~mm}$ (vertical) $\times 12.7 \mathrm{~mm}$ (horizontal) and a beam divergence of $0.07^{\circ}$ (vertical) $\times 0.18^{\circ}$ (horizontal). The scanner uses a rotating sensor scanning mechanism with 16 lasers oriented on a vertical axis. In this configuration the sensor has an angular FOV (vertical) of $\pm 15.0^{\circ}\left(30^{\circ}\right)$ and angular resolution (vertical) of $2^{\circ}$. The laser sensors spin on a horizontal axis with a rotation rate of $5 \mathrm{~Hz}-20 \mathrm{~Hz}$ to produce an angular FOV (horizontal) of $360^{\circ}$ and angular resolution (horizontal) of $0.1^{\circ}-0.4^{\circ}$. The scanner has characteristic beam divergence more suitable for an automotive application and less ideal for a mapping sensor. Nevertheless, this enables low power consumption and light weight $(830 \mathrm{~g})$ to allow its use on UAV platforms.

The remaining sensors within the integrated LiDAR sensing payload consist of a dual frequency RTKGPS/GLONASS with a lightweight antenna and inertial measurement unit (IMU). The measurements from the GPS and the IMU are synchronized with a precision internal clock, and are logged at a rate of 50 $\mathrm{Hz}$ onto an embedded computer with data storage unit, to achieve the highest possible accuracy. All these core components and wirings (except the GPS antenna) are housed inside a protective harness and fastened to the LiDAR sensor using screws. The sensor records 0.3 million laser points per second on the on-board computer and downlinks a subsampled point cloud data to the ground station in real-time, through a $5.8 \mathrm{GHz}$ long-range Wi-Fi wireless system to avoid acquisition errors. The total payload weight of the integrated LiDAR system is $1.6 \mathrm{~kg}$ and the dimensions are $16 \mathrm{~cm} \times 11.6 \mathrm{~cm} \times 11.6 \mathrm{~cm}$.

\subsubsection{System integration and aerial data acquisition}

The integrated LiDAR sensing system was mounted onto a UAV with the vertical axis of the LiDAR sensor aligned to the along track and horizontal rotating plane aligned in the across track direction of the flight trajectory. A customized coaxial rotor quadcopter 
UAV system was used to mount the integrated LiDAR sensing payload. Platform instability and vibration of the UAV platform is a critical issue for LiDAR data acquisition. High frequency vibrations produced from the rotor movements of modern UAV platforms can induce rapid movement, which is difficult for the IMU to compensate for. The quadcopter assembly provided necessary stability and the coaxial rotor configuration reduced vibration due to aerodynamic compensations, which further increased the total lift weight capacity of the UAV. Vibration from rotors was further isolated by mounting the LiDAR payload system through the use of four silicon rubber mounts. This provided sufficient physical support and compensation for the system to acquire precise point cloud data. The total system weighed under $9 \mathrm{~kg}$ and offered a flight time of around 15 minutes. The integrated UAV-LiDAR system is shown in Fig. 2 (a).

The customized coaxial quadcopter has a standalone control system based on 3DR Pixhawk2 mini flight controller. A pre-survey initialization procedure was performed, which required the UAV-LiDAR system to be powered on and then flown three times in a pattern of " 8 " to calibrate all the time-base mismatches between IMU, GPS and laser scanner. The procedure is highlighted with a blue-dashed-bounding-box in Fig. 2 (c). The UAV-LiDAR system was operated over the two test sites according to a pre-designed flight plan with a flying height of $50 \mathrm{~m}$ and speed of $5 \mathrm{~m} / \mathrm{s}$, with a transect spacing of $20 \mathrm{~m}$. In this flying configuration each test site was covered with a set of four parallel flight transects, and the entire mission was completed in two separate flights which took approximately 25 minutes in total.

\subsubsection{Raw data to point cloud}

All the raw ranging information from the laser scanner, along with the time-tagged roll, pitch, yaw and position information from the IMU and RTK-GPS/GLONASS units, was downloaded from the on-board data storage. The raw laser scanner data consists of a '.ldr' format file of the range records. The orientation and positioning data was logged on a separate file. The raw datasets were fused using a standard range transformation model (in Phoenix Aerial Spatial Fuser v3.0.5) to produce the georeferenced point cloud (in $\log$ ASCII or '.las' format). The process included correction for the centre of the IMU, RTKGPS/GLONASS unit to the laser sensor geometric translation. All 16 channels of the LiDAR were used for the generation of the point cloud. The laser footprint at nadir was $0.12 \mathrm{~m}$ along track and $0.31 \mathrm{~m}$ across track with a flying height of $50 \mathrm{~m}$. To limit laser beam divergence, laser returns with ranging values $>60 \mathrm{~m}$ were masked out in the process of point cloud generation. With the spacing between transects at $20 \mathrm{~m}$ the resultant point had a swath width of $66.3 \mathrm{~m}$ and lateral scan overlap of approximately $70 \%$. This resulted in a significant overlap of laser footprints in both along and across track directions and provided sufficient angularity to the measurements to scan further inside the tall tree canopies.

Accurate sensor localization and orientation measurements are crucial for direct georeferencing, as these parameters are not consistent throughout the UAV-LiDAR survey. To avoid error propagation into the final point cloud model due to positional or orientational uncertainties, a set of time dynamic quality threshold parameters was used. According to this criterion, for the small durations when the quality parameters were poor such as uncertainty in altitude $>10 \mathrm{~cm}$, position error $>10 \mathrm{~cm}$, number of available satellites $<9$, and differential lag $>20 \mathrm{~ms}$, the raw data to point cloud transformation was avoided.

\subsubsection{Pre-processing - point cloud sampling, segmentation and height filtering}

A LiDAR scan often contains tightly placed points that are redundant in nature. These points could be left behind due to oversampling caused by the high sampling rate of the scanner or by overlap of the point cloud from multiple transects. Such redundant points from the UAV-LiDAR point cloud were removed by sampling the data with a minimum threshold $(>0.01 \mathrm{~m})$ separation criterion. Minor errors introduced by the IMU or RTK-GPS system can cause some erroneous points to be produced in a LiDAR scan which is outside the general body of the scanned surface. A point cloud segmentation approach using connected component labelling (in CloudCompare v2) was applied with an $8^{\text {th }}$ level octree and 10,000 connected points, to identify the primary segment of the scanned surface and remove nonsurface erroneous points. Further processing was performed to filter the point cloud into ground and nonground (vegetation) classes and to calculate the heights of the vegetation points above the ground using the BCAL (BCAL LiDAR Tools for ENVI) height filtering tool (Streutker and Glenn, 2006). The parameters for canopy spacing were set at $5 \mathrm{~m}$ with a maximum vegetation height of $30 \mathrm{~m}$ in the processing step, which represented the vegetation canopy structure of Eucalyptus trees around the swamp environment. The pre-processing steps also helped filter out erroneous artefacts which would otherwise propagate to the results.

A surface point density map was produced after essential pre-processing (point cloud sampling, segmentation and height filtering) to test the quality of the resulting point cloud, i.e. to identify if the number 
of scanned points were sufficient and if a moreover uniform point distribution exists throughout the study area, which is important for generation of useful metrices from the point cloud. The surface point density map was produced by counting the number of points scanned over a regularly spaced grid of $1 \mathrm{~m}$ throughout the study area. Additionally, a histogram distribution of the surface point density was produced to identify the point density of the scan.

\subsubsection{Retrieval of LiDAR metrices}

The relative position or local relationships between a set of neighbourhood points in a LiDAR point cloud can be mathematically analyzed to derive a spatially representative surface map which is easier to use with traditional remote sensing tools. These mathematical derivatives are known as LiDAR metrices. A total of 35 LiDAR metrics related to topography, vegetation, and intensity were derived using BCAL LiDAR Tools (Table 1).

Table 1 Derived LiDAR metrices from the point cloud scan of the swamp environment.

\section{LiDAR metrices}

Topographic

Absolute roughness - The roughness (standard deviation) of all elevation points within each pixel.

Local roughness - The roughness (standard deviation) of all elevation points within each pixel after the local slope has been removed (de-trended).

Slope - The average slope of all points within each pixel in degrees.

Aspect - The aspect of the average slope of all points within each pixel in degrees from North.

Topographic solar radiation index (TRASP) - Transformation of aspect (TRASP), used by (Roberts and Cooper, 1989), is defined as $[1-\cos ((\pi / 180)($ aspect -30$))] / 2$. TRASP assigns the low value to northnortheastern aspect, and the high values to, dryer south-southwesterly slopes.

Slope cosine aspect (Slpcosasp) - Slpcosasp is calculated as slope $\times$ cosine (aspect) (Stage, 1976).

Slope Sine Aspect (Slpsinasp) - Slpsinasp is calculated as slope $\times$ sine (aspect) (Stage, 1976).

Point Density - The density of all points within each pixel.

\section{Vegetation Products}

Minimum Height - The minimum of all height points within each pixel.

Maximum Height - The maximum of all height points within each pixel.

Height Range - The difference of maximum and minimum of all height points within each pixel.

Mean Height - The average of all height points within each pixel.

Median Absolute Deviation (MAD) from Median Height - The MAD value of all height points within each pixel. $M A D=1.4826 \times$ median $(\mid$ height - median height $\mid)$.

Mean Absolute Deviation (AAD) from Mean Height - The AAD value of all height points within each pixel. $A A D=\operatorname{mean}(\mid$ height - mean height $\mid)$

Height Variance - The variance of all height points within each pixel.

Height Standard Deviation - The standard deviation of all height points within each pixel. This is also called 'absolute vegetation roughness'.

Height Skewness - The skewness of all height points within each pixel.

Height Kurtosis - The kurtosis of all height points within each pixel.

Interquartile Range (IQR) of height - The IQR of all height points within each pixel. $I Q R=Q 75-$ $Q 25$, where $\mathrm{Q}_{\mathrm{x}}$ is $\mathrm{x}^{\text {th }}$ percentile.

Height Coefficient of Variation - The coefficient of variation of all height points within each pixel.

Height Percentiles - The 5th, 10th, 25th, 50th, 75th, 90th and 95th percentiles of all height points within each pixel. 
Number of LiDAR Returns - The total number of all points within each pixel.

Number of LiDAR Vegetation Returns (nV) - The total number of all the points within each pixel that are above the specified crown threshold value (CT).

Number of LiDAR Ground Returns (nG) - The total number of all the points within each pixel that are below the specified ground threshold value (GT).

Total Vegetation Density - The percent ratio of vegetation returns and ground returns within each pixel. Density $=n V / n G * 100$.

Vegetation Cover - The percent ratio of vegetation returns $(\mathrm{nV})$ and total returns within each pixel.

Percent of Vegetation in Height Range - Percent of vegetation in height ranges 0-1 m, 1-2.5 m, 2.5$10 \mathrm{~m}, 10-20 \mathrm{~m}, 20-30 \mathrm{~m}$, and $>30 \mathrm{~m}$ within each pixel. Percent of Vegetation = Number of vegetation returns in the range/Total vegetation returns

Canopy Relief Ratio - Canopy relief ratio of points within each pixel. Canopy relief ratio $=$ $\left(\left(H_{\text {mean }}-H_{\text {min }}\right)\right) /\left(\left(H_{\text {max }}-H_{\text {min }}\right)\right)$.

Texture of Heights - Texture of height of points within each pixel, i.e. standard deviation of height above ground threshold and height below crown threshold.

Foliage Height Diversity (FHD) - is denoted by $F H D=-\sum p_{i} \ln \left(p_{i}\right)$ where pi is the proportion of the number of LiDAR returns in the $\mathrm{i}^{\text {th }}$ layer to the sum of LiDAR points of all the layers (using all points).

Foliage Height Diversity above ground threshold (FHD $($ GT $)$ - Points above ground-FHD calculated only using points above GT.

\section{Intensity}

Minimum Intensity - The point with the minimum intensity value within each pixel.

Maximum Intensity - The point with the maximum intensity value within each pixel.

Mean Intensity - The mean intensity of all points within each pixel.

Standard Deviation Intensity - The standard deviation of intensity value of all points within each pixel.

Generation of LiDAR metrices from highly dense point cloud acquired from UAV-LiDAR is a computationally intense and time-consuming process, particularly in the absence of a graphics processing unit (GPU) based multi-core parallel processing capabilities with BCAL. Therefore, LiDAR metrices (Table 1) generation was processed at a grid size or pixel resolution of $10 \mathrm{~cm}$ to limit excessive processing time. The metrices were derived with a ground threshold of $10 \mathrm{~cm}$ and a crown threshold of $20 \mathrm{~cm}$. A bin height of $1 \mathrm{~cm}$ was used for the computation of foliage height density (FHD) parameters.

\subsubsection{Extracting Canopy height model from LiDAR data}

A bare earth digital elevation model (DEM) at $10 \mathrm{~cm}$ resolution was produced using the LiDAR point cloud by only considering the last returns in BCAL. The process assigns the minimum altitude value of the last return points within each grid or pixel size of $10 \mathrm{~cm}$. The model approximates a DEM surface by interpolation in dense areas with the absence of correct ground level measurements due to dense canopies. A digital surface model (DSM) was also computed by using the first returns of LiDAR point cloud, and assigning the maximum altitude value of any point within the $10 \mathrm{~cm}$ grid to the corresponding pixel value of the DSM. The canopies of shrub-type swamp vegetation are often fragile or tilted (due to wind), which often does not produce sufficient first return measurements for correct canopy height measurements. A $5 \times 5$ local maximum filter was applied on the DSM to assign the maximum height within an area of $50 \mathrm{~cm}$ by $50 \mathrm{~cm}$ to the central pixel. A canopy height model (CHM) was then measured by subtracting the DEM from the filtered DSM. The accuracy of the canopy height model was validated against the field based measurements of canopy height of shrub-type vegetation using coefficient of determination $\left(\mathrm{R}^{2}\right)$ statistics (Cameron and Windmeijer, 1997).

\subsubsection{Classification of LiDAR data for mapping}

The stacked LiDAR metrices was used to differentiate the different types of swamp vegetation classes. A set of seven supervised classifiers such as parallelepiped (PP), maximum likelihood (ML), minimum distance (MD), Mahalanobis distance (MHD), spectral angle 
mapper (SAM), spectral information divergence (SID), and support vector machine (SVM) were used from ENVI (Exelis Inc., Harris Corporation, Boulder, Colorado, United States) to demonstrate the ability of the different datasets in the classification process and cross-validation. To mutually evaluate the classifiers a standard 'null' parameter setting was used for each classifier i.e., no standard deviation threshold from mean was used for PP, MD and MHD, no probability threshold was used for ML, no maximum threshold angle for the SAM, and no maximum divergence angle was used for SID. The SVM classifier out of the set of seven classifiers is based on machine learning, and as such, was computationally intensive. However, the objective here was not to identify an efficient and robust classification workflow but to evaluate the potential of LiDAR data in mapping sensitive vegetation communities with well-established classification methodologies. A standard parameter setting using a radial basis function with a kernel gamma function of 0.167 , penalty parameter of 100 and pyramid level of 5 was used.

The 35 LiDAR metrices (Table 1) were first stacked to produce a composite multi-dimensional dataset. However, all the derived LiDAR metrices do not necessarily contain useful information for classification. Therefore, two data dimensionality reduction techniques - principal component analysis (PCA) (Richards and Richards, 1999) and independent component analysis (ICA) (Hyvärinen and Oja, 2000) were used to condense the information content of 35 LiDAR metrics. This step essentially condensed the useful information from all the 35 metrices to the initial layers of the stacked dataset. The first 15 LiDAR metrices with high information content were then selected from the stacked dataset, where the eigenvalues $>0.2$. A $3 \times 3$ enhanced frost filter (Lopes et al., 1990) was used to adaptively average pixel values in homogenous clusters with a coefficient of variation, $\mathrm{C}_{\mathrm{u}}=0.523$, and an impulse response convolution kernel for heterogeneous clusters with a maximum coefficient of variation, $\mathrm{C}_{\max }=1.732$. At this stage, the dimensionally reduced and filtered 15 metrices composite data can undergo a classification operation similar to the multispectral and hyperspectral datasets.

A total of 320 ground truth measurements were collected for shrub-type swamp vegetation through a rigorous field survey, the vegetation class for each point was geospatialised as a field attribute. A buffer with a radius of $5 \mathrm{~cm}$ was created around each of the 320 measurements to produce an equivalent ground truth polygon for each measurement. An additional 128 ground truth polygons were also created through visual interpretation of high-resolution optical data maps. Ground truth samples for bush or tree-type vegetation classes such as Dagger hakea, Heath-leaved banksia, Black sheoak, and Eucalyptus trees were primarily acquired using this approach. The sampled groundbased (320) and image-based (128) polygons were randomly divided into $1: 1$ mutually exclusive sets of training and test samples, i.e. 160 ground and 64 image-based polygons for each training and test group. The ground truth training set was used to train the classifiers, and the test samples were used to compute the overall accuracy (OA), kappa ( $\kappa)$ and confusion matrix to evaluate the classification accuracies. All the seven classifiers were applied to the datasets at $10 \mathrm{~cm}$ resolution, and the overall accuracy (OA) and kappa ( $\kappa)$ values were tabulated. Classification maps based on the best classifier performance and the corresponding confusion matrix were produced.

\section{Results}

\subsection{Geometric quality assessment for UAV- LiDAR}

The UAV-LiDAR system and the raw data to point cloud processing segment produced a well georeferenced point cloud. The accuracy of the UAVLiDAR metrices obtained through the processing chain was tested against referenced optical maps. The point cloud achieved an average error of $10.4 \mathrm{~cm}$, which was deemed sufficient for further environmental monitoring applications. For the study area, a UAVLiDAR point cloud with a surface height profile for a portion of the study area is shown in Fig. 3.

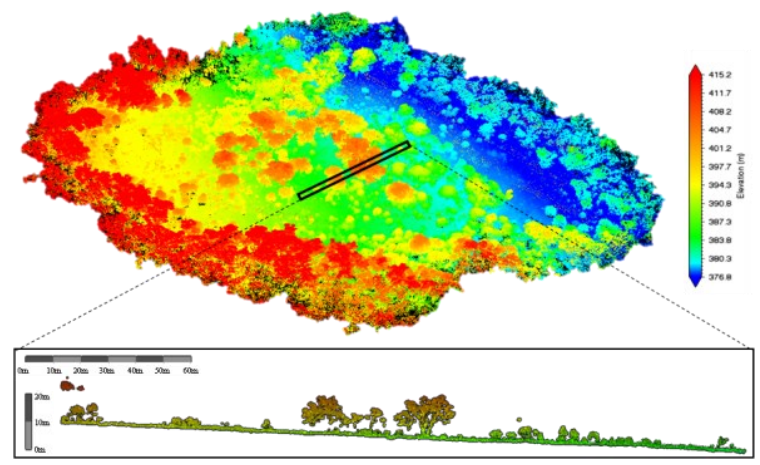

Fig. 3 Point cloud obtained through UAV-LiDAR survey of the study area with a cross-section view of the surface height profile for a portion the swamp. 


\subsection{Surface point density and effect of pre- processing}

An uniform surface density map is essential for accurate processing and generating higher order products from the LiDAR point cloud, such as LiDAR metrices. The programmed flight plan of the LiDAR scan produced a more uniform distribution of points. The point cloud was further processed to filter out redundant points which improved the uniformity of the point cloud. This facilitates a moreover uniform distribution of surface point density, which is useful for avoiding density induced bias in the computation of LiDAR metrices. The calculated surface point density map for the study area is shown in Fig. 4 (a). A high density of point cloud is also essential for accurate fine-scale mapping applications. A histogram of the surface point density plot is shown in Fig. 4(b).

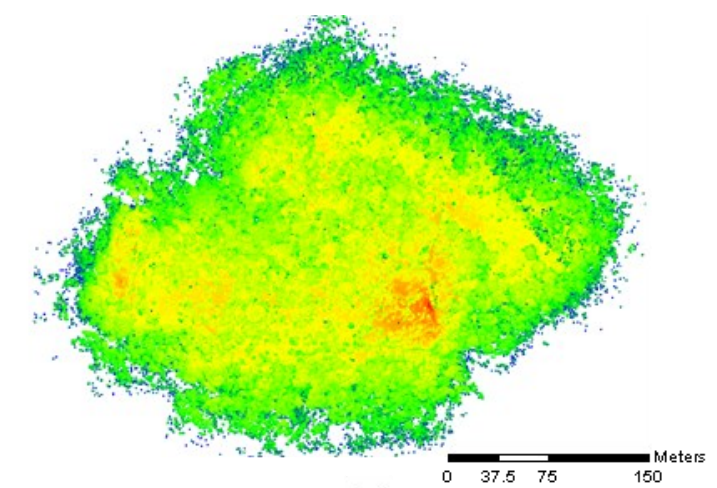

(a)

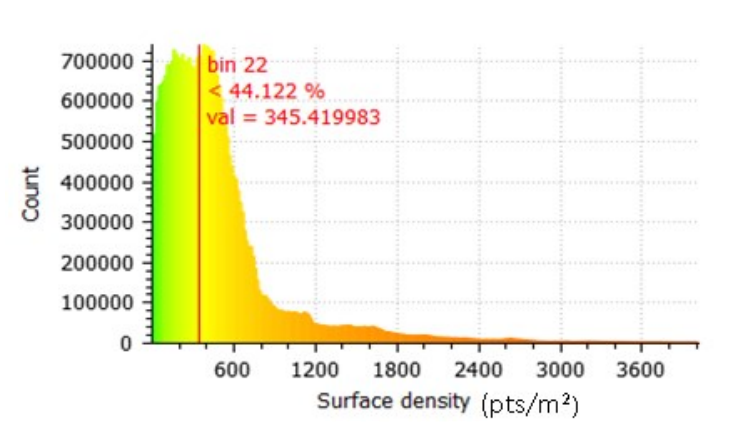

(b)

Fig. 4 (a) Surface point density map obtained through UAV-LiDAR survey of the study area and (b) histogram distribution of the surface point density - also showing the colour scale.

The scanned and pre-processed point cloud achieved a very high surface point density, with a distribution of $345 \mathrm{pts} / \mathrm{m}^{2}$. This distribution of point cloud density is significantly higher than what was traditionally achieved through airborne surveys $\left(\sim 20-40 \mathrm{pts} / \mathrm{m}^{2}\right)$. This means UAV-LiDAR metrices can be produced with a significant level of accuracy and detail, which is beneficial to identify and distinguish the complex distribution of vegetation communities in a diverse ecosystem area such as swamps. Furthermore, high point density increases the likelihood of more points being recovered from under the canopies, which is essential to produce an accurate topographical surface model to identify any deformation induced from underground longwall mining. This indicates the potential benefit of using a UAV-LiDAR system in environmental applications requiring fine-scale mapping.

The pre-processing steps to filter the point cloud are also essential to avoid propagation to errors in the LiDAR metrices. A synoptic overview of the preprocessed point cloud is shown in Fig. 5. A false coloured composite of the processed LiDAR metrices (kurtosis, maximum vegetation height and coefficient of variation) for a spatially subset region is shown in Fig. 5 (a) without any pre-processing and in Fig. 5 (b) with proper pre-processing. The comparison demonstrated the efficacy of the preprocessing phase to avoid erroneous artefacts in the processed false coloured composite of the LiDAR metrics, marked in white ovals; such artefacts are removed with pre-processing.
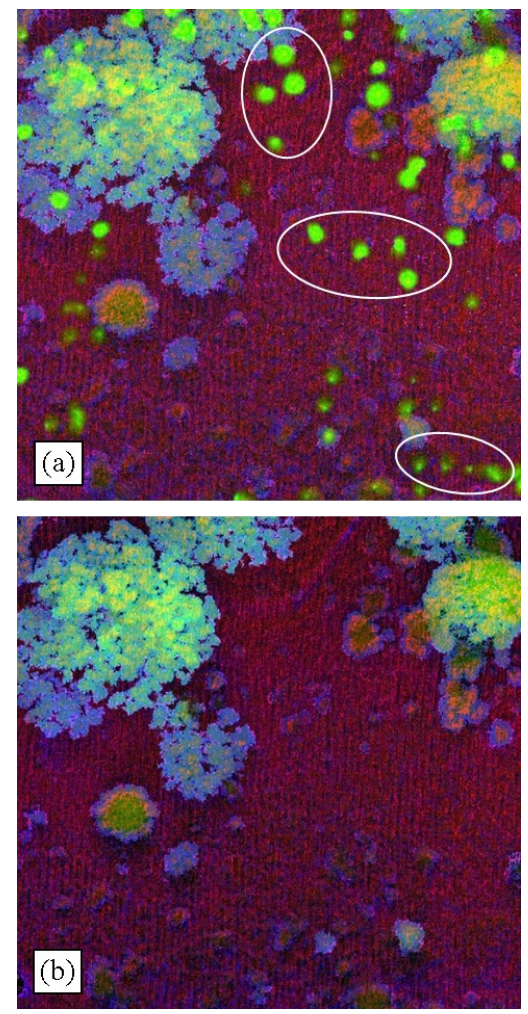

Fig. 5 (a) Pre-processed point cloud (colourized as per elevation), and false colour composite (kurtosis, maximum vegetation height and coefficient of variation) of LiDAR matrices for a subset area: (b) without pre-processing and (c) with pre-processing. 


\subsection{LiDAR metrices}

The point cloud from the UAV-LiDAR survey was processed to produce a total of 35 LiDAR metrices related to topography, vegetation structure and intensity. The computed metrices are equivalent to raster products, which can differentiate different vegetation species and classes using a classical classification workflows and provide information related to swamp vegetation conditions. A few of the
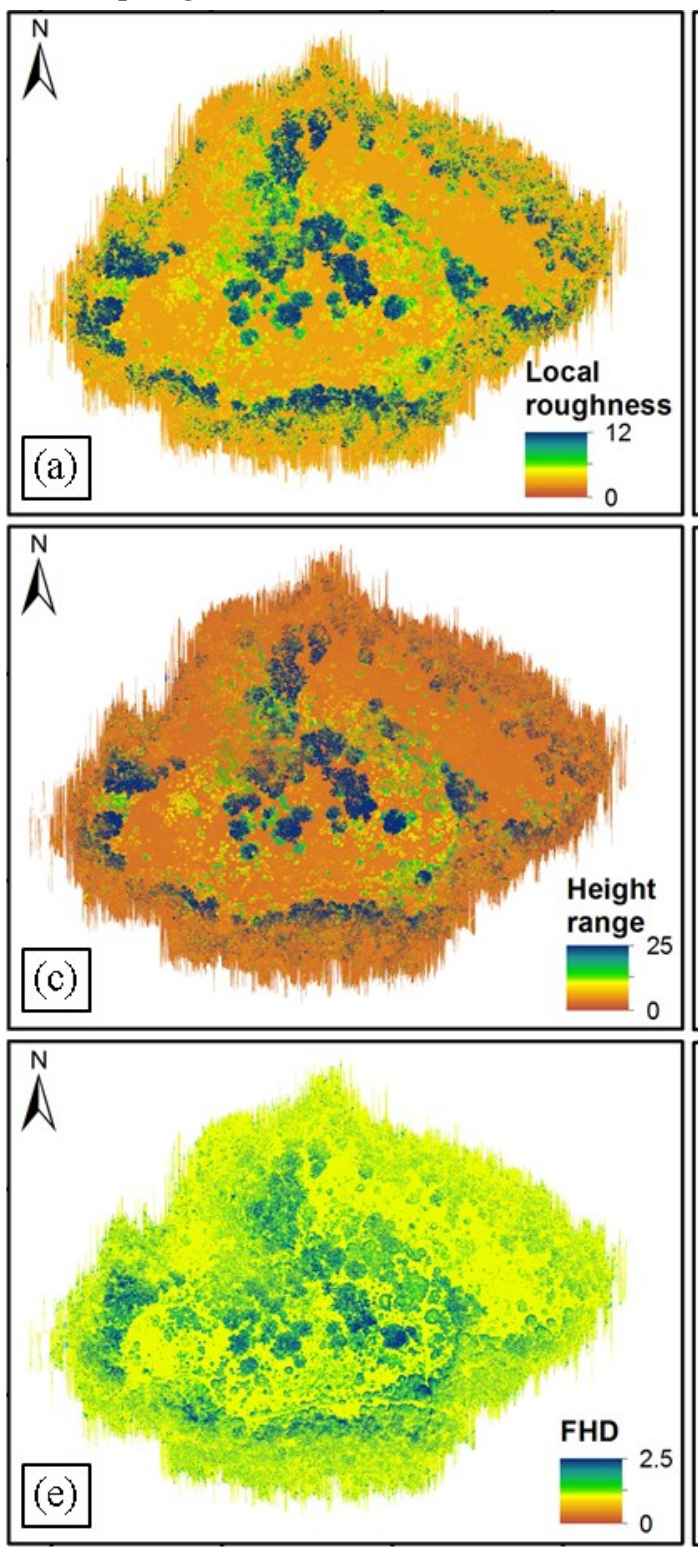

$0 \quad 60 \quad 120$ 240

Meters

selected maps of LiDAR metrices such as local roughness, slope cosine aspect (Slpcosasp), height range, vegetation cover, foliage height density (FHD) and mean intensity are shown in Fig. 6. The swamp area can be visually distinguished from the surrounding terrestrial type Eucalyptus trees without further processing as well. This shows the potential of a highdensity of point cloud obtained through the UAVLiDAR survey.
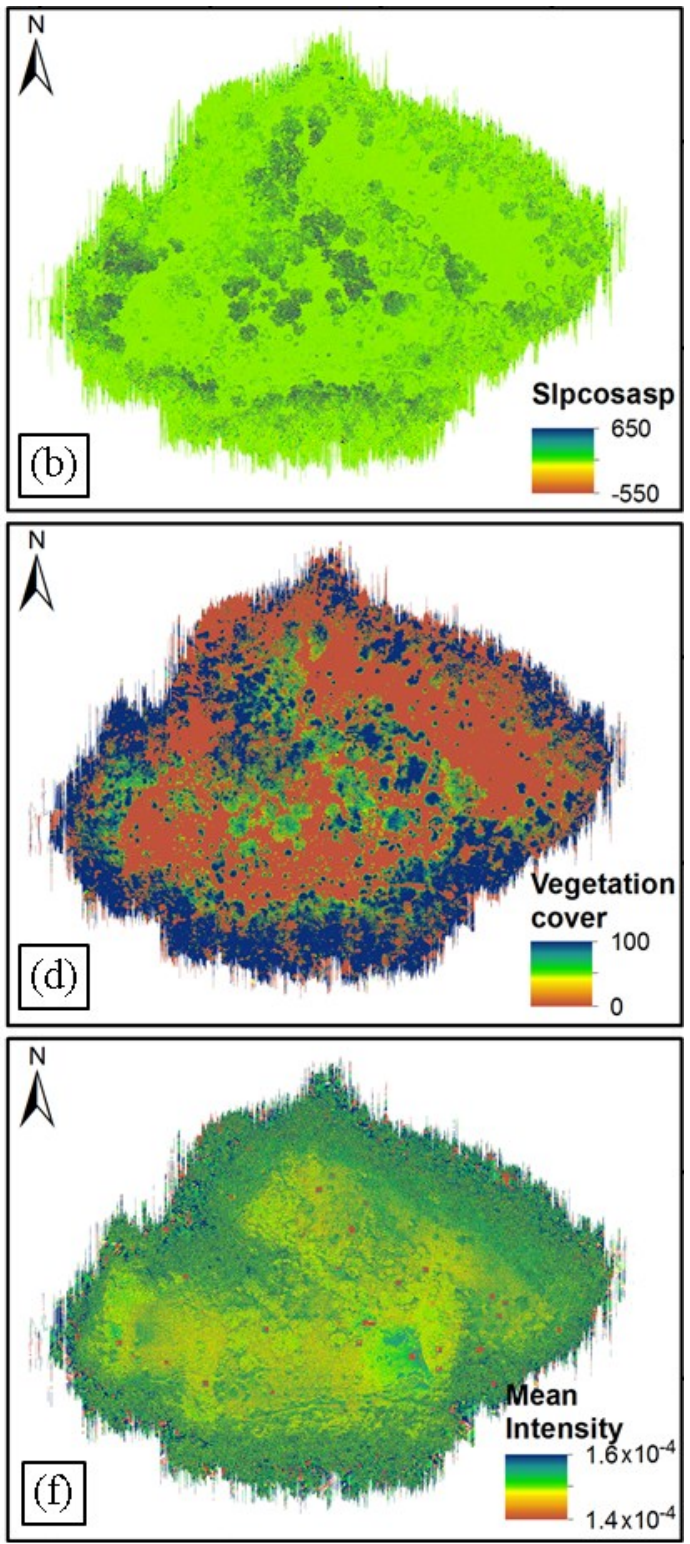

Fig. 6 LiDAR metrices maps of (a) local roughness, (b) slope cosine aspect (Slpcosasp), (c) height range, (d) vegetation cover, (e) foliage height density (FHD) and (f) mean intensity.

\subsection{Canopy height model}

The UAV-LiDAR point cloud was processed to produce a canopy height model (CHM) of the swamp environment. A CHM is useful for characterization of the extent of an upland swamp environment by differentiating the low-lying peat swamp vegetation from the surrounding terrestrial vegetation such as Eucalyptus trees (Jenkins and Frazier, 2010). A colour scaled synoptic view of the CHM for the two swamp 
sites in the study area is shown in Fig. 7 (a) and a textured three-dimensional view is shown in Fig. 7 (b). The accuracy of the CHM was analyzed with the ground truth values of canopy height measurements for shrub-type swamp vegetation cover. The overall $\mathrm{R}^{2}$ accuracy was found to be approximately 0.76 , which was deemed sufficient for the type of vegetation cover. The computation of accurate CHM for shrub-type vegetation is relatively difficult compared to tree-type vegetation cover. This depends on two factors: the small and fragile nature of shrub-type vegetation canopies, and the footprint size of the laser. Under these scenarios the accuracy of models could be improved by improving the beam width of the internal laser sensor in the UAV-LiDAR system or by incorporating machine learning methods to perform parametric transformation of the coarse CHM product using reference tie point measurements.
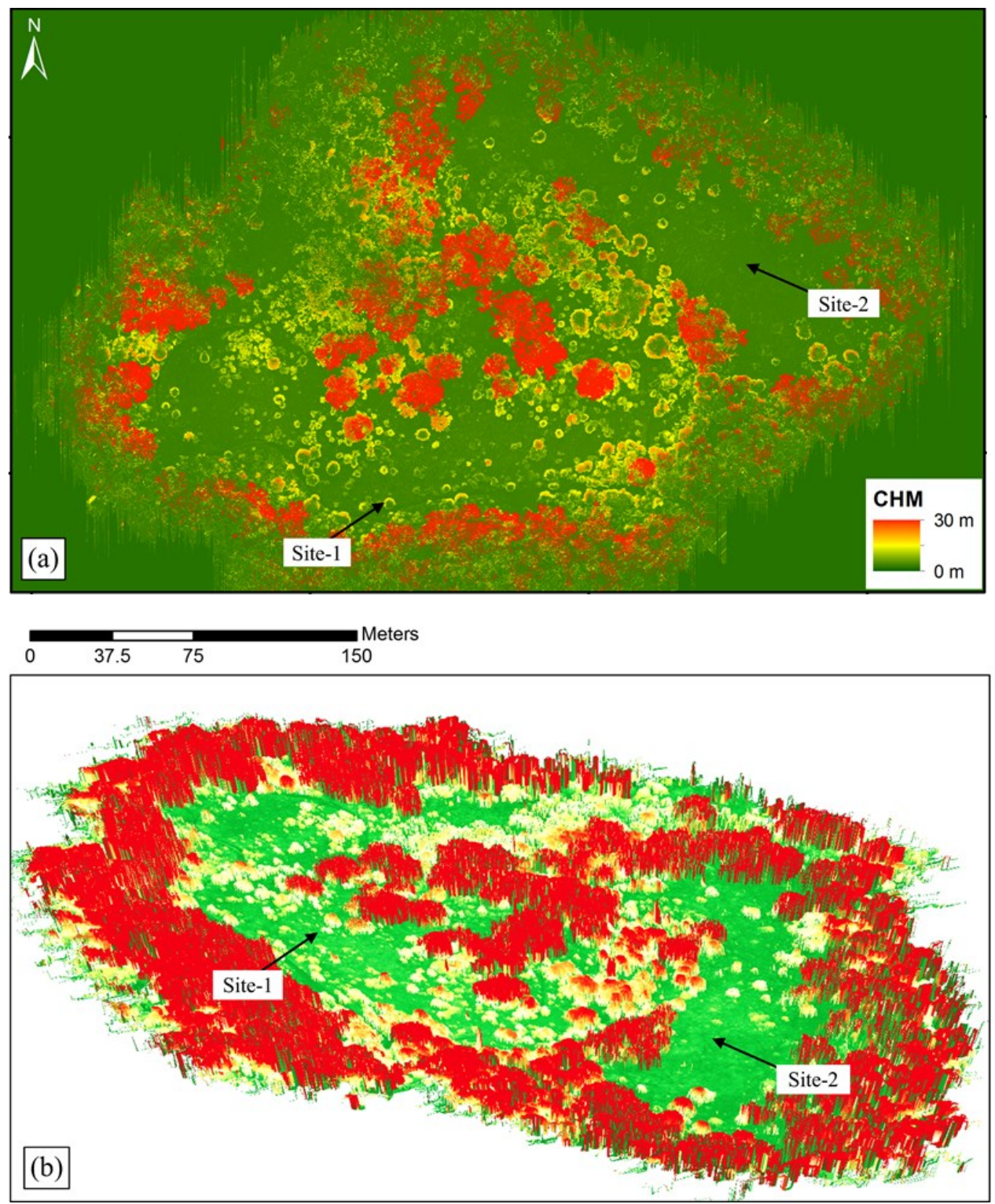

Fig. 7 Canopy height model of the study area: (a) synoptic view and (b) textured three-dimensional view.

\subsection{Classification of vegetation communities}

Data collected using UAV-LiDAR system was evaluated using a classification based approach. The respective datasets were collected for both swamp site1 and site-2. A total of eight vegetation classes as described in Section 2.1 were present in swamp site-1 and site- 2 , these eight vegetation classes were used to operate the classification based evaluation, i.e. Dagger hakea, Grass tree, Heath-leaved banksia, Black sheoak, Bracken fern Eucalyptus tree, Pouched coral fern and Sedgeland complex. Additionally, some portion of the acquired image area comprised of no-vegetation cover and was treated as a separate 'Bare earth' class, i.e., a total of nine classification classes. The UAV-LiDAR metrices were dimensionally reduced using PCA and 
ICA, both of which were used for comparative analysis. The overall accuracy and kappa coefficient of the methods are listed in Table 2. The ICA(LiDAR) marginally outperformed the PCA(LiDAR) with most classifiers, with the exception of PP and SID. The best classification result was produced by combining the ICA(LiDAR) data with SVM classifier, which produced an overall accuracy of $73.42 \%$ and a kappa coefficient of 0.64 .

Table 2 Accuracy assessment for the classification of UAV-LiDAR data.

\begin{tabular}{lcccc}
\hline & \multicolumn{1}{c}{$\boldsymbol{P C A}(\mathbf{L i D} \boldsymbol{A R})$} & \multicolumn{2}{c}{ ICA(LiDAR) } \\
\cline { 2 - 5 } Classifier & $\begin{array}{c}\text { Overall } \\
\text { accuracy } \\
(\%)\end{array}$ & Kappa & $\begin{array}{c}\text { Overall } \\
\text { accuracy }\end{array}$ & Kappa \\
\hline Parallelepiped (PP) & $\mathbf{1 8 . 4 9}$ & $\mathbf{0 . 1 3}$ & 5.91 & 0.04 \\
Maximum Likelihood (ML) & 58.77 & 0.49 & $\mathbf{6 0 . 0 0}$ & $\mathbf{0 . 5 1}$ \\
Minimum Distance (MD) & 43.16 & 0.33 & $\mathbf{4 5 . 4 9}$ & $\mathbf{0 . 3 5}$ \\
Mahalanobis Distance (MHD) & 48.02 & 0.37 & $\mathbf{4 8 . 3 1}$ & $\mathbf{0 . 3 8}$ \\
Spectral Angle Mapper (SAM) & 43.73 & 0.33 & $\mathbf{4 5 . 4 9}$ & $\mathbf{0 . 3 5}$ \\
Spectral Information Divergence (SID) & $\mathbf{4 6 . 8 4}$ & $\mathbf{0 . 3 7}$ & 32.08 & 0.23 \\
Support Vector Machine (SVM) & 73.37 & 0.64 & $\mathbf{7 3 . 4 2 *}$ & $\mathbf{0 . 6 4 *}$ \\
\hline best result & & & &
\end{tabular}

The producer's and user's accuracy for each class with the best classification method is shown in Table 3. The classification accuracy was high for bare earth, Dagger hakea, Black sheoak, Eucalyptus trees and Sedgeland complex. However, the accuracy for Grass tree, Heath-leaved banksia, Bracken fern and Pouched coral fern was exceptionally low. The low accuracy with these classes for LiDAR is not surprising since the structural characteristics of these species are indistinguishable through LiDAR point cloud. The accuracy was high for tall terrestrial trees such as Black sheoak and Eucalyptus, which most importantly improved classification accuracy of the Sedgeland complex class. The classification maps for and ICA(LiDAR) based approach using SVM classifier is shown in Fig. 8.

Table 3 Class wise accuracy of best classification method - ICA(LiDAR) with support vector machine.

\begin{tabular}{lcc}
\hline Class & Producer's accuracy (\%) & User's accuracy (\%) \\
\hline Bare earth & 78.12 & 82.88 \\
Dagger hakea & 99.17 & 92.07 \\
Grass tree & 16.95 & 32.51 \\
Heath-leaved banksia & 11.02 & 62.22 \\
Black sheoak & 87.35 & 73.18 \\
Bracken fern & 40.23 & 50.75 \\
Eucalyptus tree & 80.67 & 91.25 \\
Pouched coral fern & 58.68 & 52.45 \\
Sedgeland complex & 94.85 & 64.06 \\
\hline
\end{tabular}




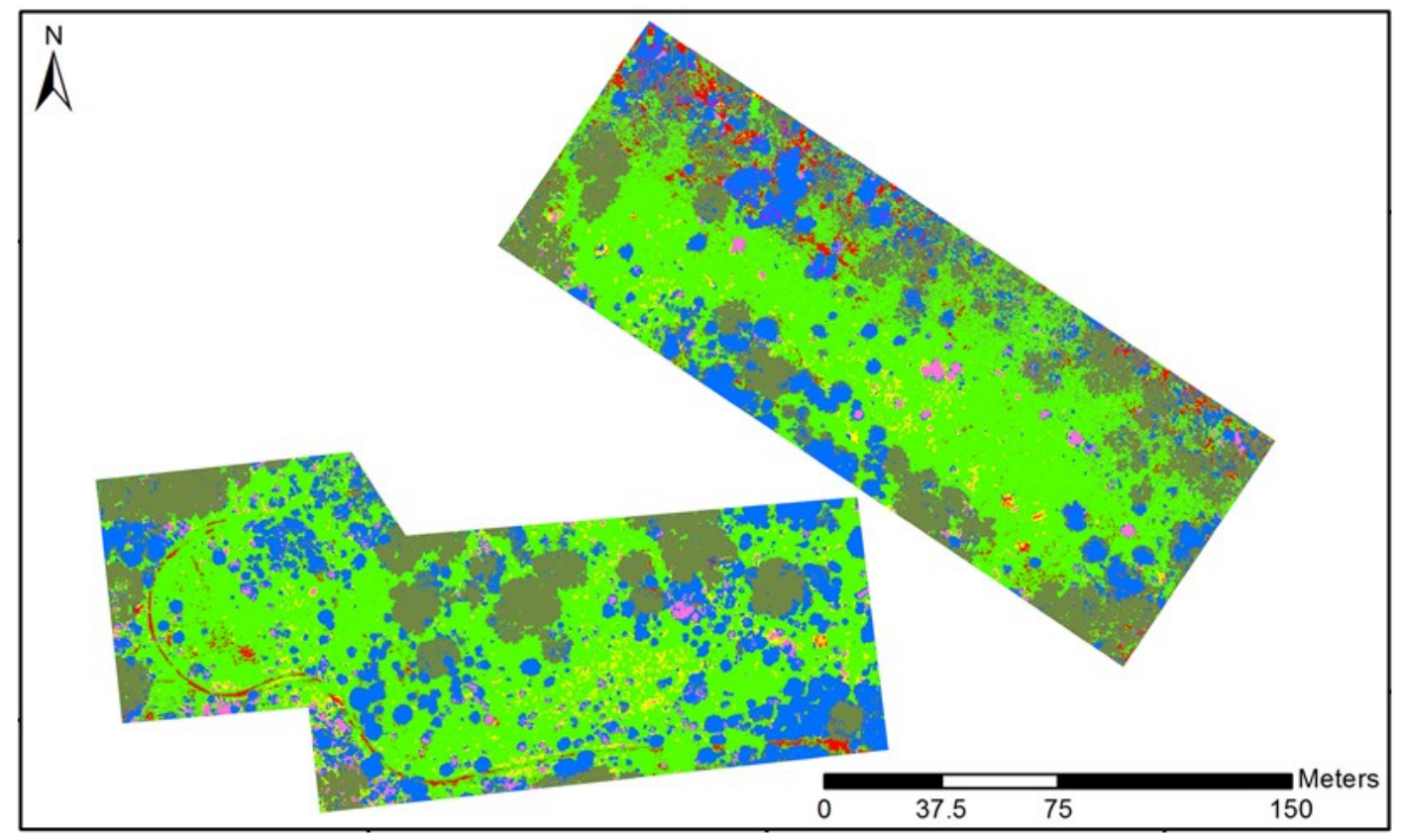

\section{Legend}
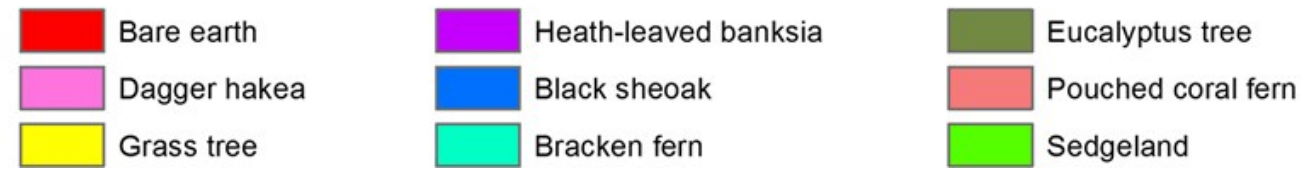

Fig. 8 Classification map of swamp site-1 and site-2 vegetation classes and species produced with (a) UAVhyperspectral and (b) UAV-LiDAR data, using support vector machine classifier.

\section{Discussion}

\subsection{Integration of UAV-LiDAR system}

The UAV-LiDAR system were developed through sensor and platform integration, including aspects of sensor calibration, sensor operation, orientation, mission planning and data acquisition. The installation of a LiDAR sensor on the UAV required thorough consideration of several aspects of sensor parameters such as laser range, GPS and IMU accuracy, beam width, scanning mechanism, field-of-view and angle of scanning. The mission planning focused on these sensor parameters to obtain an accurate point cloud with high surface point density, which required a design of a suitable calibration loop, and flight paths with significant overlap of laser footprints in both along and across track directions. Attention and diligence to these aspects of system integration and operation were essential to seamlessly retrieving accurate and effective data products. Overall, these considerations and innovative tuning towards various design and integration phases were essential in mine environmental monitoring, and other applications requiring accurate thematic mapping with UAVs such as agriculture, forestry.

\subsection{High-resolution point cloud from UAV- LiDAR}

Acquisition of high-density point cloud is essential for detailed 3D imaging of the environment. Several factors such as speed of UAV-LiDAR system during scanning, distance from the target or flying height, spacing between transects and across track scan width influences the density of points collected during a scan. Furthermore, quality influencing factors such as uncertainty in altitude, position error, number of available satellites, and differential lag need to be controlled to obtain well-registered point cloud data. A workflow was devised through the combination of a set of different software and processing solutions to effectively convert raw LiDAR return, position, orientation and quality information to a point cloud with high surface density, to pre-process the point cloud to remove redundant points and IMU induced errors, and to prepare the point cloud for further processing. The geometric accuracy of the integrated UAV-LiDAR system is crucial for fine-scale monitoring and mapping applications. A dedicated geometric quality assessment exercise confirmed the high accuracy of the system. The system produced a very high-density point cloud in the complex swamp 
environment, which was useful for the differentiation of several vegetation types.

\subsection{Derived LiDAR metrices}

The complex assemblage of swamp vegetation species and communities was studied using a UAV-based LiDAR system. A total of 35 LiDAR metrices related to topography, vegetation structure and intensity were produced. The computed metrices are raster products similar to hyperspectral indices, which can be used to differentiate different vegetation species and communities using a classical classification workflow and also provide useful information on swamp vegetation condition. The vegetation indices and selected LiDAR metrices produced through UAV survey were validated and cross-validated against biophysical parameters such as leaf area index (LAI) and canopy height model (CHM).

Deriving LiDAR metrices from high-density point cloud is a computationally-intensive process. To provide an estimate, a spatial subset of the point cloud of approximately one tenth the size of the total point cloud at a point density of $345 \mathrm{pts} / \mathrm{m}^{2}$ took over a week of processing to produce LiDAR metrices at $2 \mathrm{~cm}$ of resolution. This order of computational inefficiency is limiting for routine environmental monitoring operations. Nevertheless, LiDAR metices could be generated at lower spatial resolutions $(>10 \mathrm{~cm})$ relatively easily. However, reconstruction of a multicore parallel computation pipeline for the generation of LiDAR metrices, could be a future scope for fine-scale mapping applications using UAV-LiDAR.

\subsection{Monitoring vegetation communities using UAV-LiDAR}

Satellite based spectral monitoring has been used to differentiate bog from surrounding surface cover, such as sedge lands or grasslands, and identify any gain or loss in woody vegetation (Lechner et al., 2012). Such methods are effective in monitoring swamps, if the phenology of the vegetation is documented, and especially, if changes in phenology due to presence or absence of water is known (CoA, 2014). Natural fluctuations in variability over time are indicative of growth rates and phenology which could be analyzed using remote sensing images of wet and dry seasons. Existing phenology products such as Australian Phenology Product available from Terrestrial Ecosystem Research Network (TERN) is coarse $(5.6 \mathrm{~km})$ in resolution and limited to regional level ecosystem modelling. THPSS sites represent small patches of the diverse ecosystem, which limits the applicability of readily available regional phenology products. Ground-based phenological survey products could not be found for the study area. Through the development of appropriate UAV-based sensing systems, this study acts as a foundation to generate fine-scale vegetation maps through UAV-based remote sensing surveys in future.

Within the Sedgeland-complex class the composition of species varies from one region in the swamps to another, and these subtle variations result in homogenous to heterogeneous conglomerations of target species as different sub-categories. Banerjee et al. (2017) identified the challenges in classifying these sub-categories of Sedgeland complex as distinct classes, due to the scale at which these species are mixed together, resulting in complex spectral and structural intermixing. However, it was essential to treat these sub-categories distinctly for biophysical parameter retrieval. Hence, the species composition mapping through further investigations has been performed using UAV-hyperspectral, however, only spectral information was insufficient in accurately mapping complex assemblages of species in swamp environments (Banerjee et al., 2020). Therefore, this study investigated the potential of LiDAR as a structural modality in mapping vegetation communities in diverse ecosystems. Additionally, the classification maps produced with the UAV-LiDAR system are less prone to shadow effects than optical and hyperspectral datasets. In future, a fusion based approaches between UAV based hyperspectral and LiDAR datasets could be valuable in accurately mapping complex vegetation communities.

\section{Conclusion}

Traditional satellite and airbone remote sensing has been a widely used tool in scientific research and environmental monitoring at global and regional levels. Its adoption in fine-scale monitoring and mapping related applications has been challenging due to limited spatial resolution, atmospheric noise and cloud cover. The advent of near earth imaging systems such as UAV-LiDAR has offered the potential in detail mapping and monitoring of landcover units. Although these systems are popular in allied disciplines such as agriculture and forestry, their application for monitoring complex ecosystems and environments such as swamps has been limited. This is partly due to the absence of an existing methodological framework and challenges in using UAV-based sensor systems in these sensitive and complex environments. The primary objective of this research was to develop a functional UAV-based LiDAR sensing system, including a workflow to generate accurate datasets and products for environmental monitoring applications. A critical review was undertaken to identify the current state and limitations of UAV-based LiDAR technologies for mine environmental monitoring 
research. Several issues that make effective use of these technologies challenging for seamless data generation and processing were identified and resolved, leading to the classification of complex vegetation communities in sensitive ecosystems. The technology and methodology demonstrated herein would be potentially valuable in identifying changing conditions or the health of ecosystems.

Author Contributions: B.P.B. conceived the experiment, conducted the experiments, performed data analysis and drafted the original manuscript. S.R. conducted the project administration, supervision and manuscript review and editing. All authors reviewed the manuscript. All authors have read and agreed to the published version of the manuscript.

\section{Statements and Declarations}

Funding: No funding was received to assist with the preparation of this manuscript.

Financial interests: The authors declare they have no financial interests.

Conflicts of Interest: The authors declare no conflicts of interest.

\section{Reference}

Akcil A, Koldas S (2006) Acid Mine Drainage (AMD): causes, treatment and case studies. Journal of Cleaner Production 14 (12):1139-1145

Banerjee BP, Raval S (2021) A Particle Swarm Optimization Based Approach to Pre-Tune Programmable Hyperspectral Sensors. Remote Sensing 13 (16):3295

Banerjee BP, Raval S, Cullen PJ (2017) Highresolution mapping of upland swamp vegetation using an unmanned aerial vehicle-hyperspectral system. Journal of Spectral Imaging 6 (1):a6

Banerjee BP, Raval S, Cullen PJ (2020) UAVhyperspectral imaging of spectrally complex environments. International Journal of Remote Sensing 41

(11):4136-4159.

doi:10.1080/01431161.2020.1714771

Banerjee BP, Raval S, Cullen PJ, Shen XJ (2018) Mapping of complex vegetation communities and species using uav-lidar metrics and high-resolution optical data. Paper presented at the 38th annual symposium of IEEE International Geoscience and Remote Sensing Symposium, Valencia, Spain,

Cameron AC, Windmeijer FA (1997) An R-squared measure of goodness of fit for some common nonlinear regression models. Journal of econometrics 77 (2):329342
Chang J, Hu T, Liu X, Ren X (2018) Construction of green infrastructure in coal-resource based city: a case study in Xuzhou urban area. International Journal of Coal Science \& Technology 5 (1):92-104. doi:10.1007/s40789-018-0191-8

CoA (2014) Temperate Highland Peat Swamps on Sandstone: ecological characteristics, sensitivities to change, and monitoring and reporting technique. Independent Expert Scientific Committee on Coal Seam Gas and Large Coal Mining Development, Department of the Environment, Australian Government,

Colomina I, Molina P (2014) Unmanned aerial systems for photogrammetry and remote sensing: A review. ISPRS Journal of Photogrammetry and Remote Sensing $92 \quad$ (0):79-97. doi:http://dx.doi.org/10.1016/j.isprsjprs.2014.02.013

Congalton RG (1991) A Review of Assessing the Accuracy of Classifications of Remotely Sensed Data. Remote Sensing of Environment 37 (1):35-46. doi:Doi 10.1016/0034-4257(91)90048-B

Englhart S, Jubanski J, Siegert F (2013) Quantifying dynamics in tropical peat swamp forest biomass with multi-temporal LiDAR datasets. Remote Sensing 5 (5):2368-2388

Gandaseca S, John S, Haruna Ahmed O, Muhamad N (2009) Vegetation assessment of peat swamp forest using remote sensing. American Journal of Agricultural and Biological Sciences 4 (2):167-172

Greene R, Timms W, Rengasamy P, Arshad M, Cresswell R (2016) Soil and aquifer salinization: Toward an integrated approach for salinity management of groundwater. In: Integrated Groundwater Management. Springer, pp 377-412

Hyvärinen A, Oja E (2000) Independent component analysis: algorithms and applications. Neural networks $13(4): 411-430$

Jaakkola A, Hyyppä J, Kukko A, Yu X, Kaartinen H, Lehtomäki M, Lin Y (2010) A low-cost multi-sensoral mobile mapping system and its feasibility for tree measurements. ISPRS Journal of Photogrammetry and Remote Sensing $65 \quad$ (6):514-522. doi:10.1016/j.isprsjprs.2010.08.002

Jenkins RB, Frazier PS (2010) High-resolution remote sensing of upland swamp boundaries and vegetation for baseline mapping and monitoring. Wetlands 30 (3):531-540. doi:10.1007/s13157-010-0059-1

Lechner A, Fletchera A, Johansen K, Erskinea P (2012) Characterising Upland Swamps Using Object-based Classification Methods and Hyper-spatial Resolution Imagery Derived from an Unmanned Aerial Vehicle. ISPRS Annals of the Photogrammetry, Remote Sensing and Spatial Information Sciences I-4:101-106 
Lin Y, Hyyppa J, Jaakkola A (2011) Mini-UAV-borne LIDAR for fine-scale mapping. IEEE Geosci Remote Sens Lett 8 (3):426-430

Lopes A, Touzi R, Nezry E (1990) Adaptive speckle filters and scene heterogeneity. IEEE transactions on Geoscience and Remote Sensing 28 (6):992-1000

The Native Vegetation of the Woronora, O'Hares and Metropolitan Catchments (2003) NSW National Parks and Wildlife Service (NPWS)

NSWDP (2008) Impacts of Underground Coal Mining on Natural Features in the Southern Coalfield: Strategic Review. Sydney, NSW, Australia

Opitz J, Timms W (2016) Mine water discharge quality-a review of classification frameworks.

Ren H, Zhao Y, Xiao W, Hu Z (2019) A review of UAV monitoring in mining areas: current status and future perspectives. International Journal of Coal Science \& Technology 6 (3):320-333. doi:10.1007/s40789-019-00264-5

Richards JA, Richards J (1999) Remote sensing digital image analysis, vol 3. Springer,

Setiawan Y, Pawitan H, Prasetyo L, Permatasari P Monitoring tropical peatland ecosystem in regional scale using multi-temporal MODIS data: Present possibilities and future challenges. In: IOP Conference Series: Earth and Environmental Science, 2017. vol 1. IOP Publishing, p 012052

Setiawan Y, Pawitan H, Prasetyo LB, Parlindungan M, Permatasari PA (2016) TEMPORAL VEGETATION DYNAMICS IN PEAT SWAMP AREA USING MODIS TIME-SERIES IMAGERY: A MONITORING APPROACH OF HIGH-SENSITIVE ECOSYSTEM IN REGIONAL SCALE. Geoplanning: Journal of Geomatics and Planning 3 (2):137-146

Strecha C, Fletcher A, Lechner A, Erskine P, Fua P (2012) Developing species specific vegetation maps using multi-spectral hyperspatial imagery from unmanned aerial vehicles. ISPRS Annals of the Photogrammetry, Remote Sensing and Spatial Information Sciences 3:311-316

Streutker DR, Glenn NF (2006) LiDAR measurement of sagebrush steppe vegetation heights. Remote Sensing of Environment 102 (1):135-145

Vervoort A (2021) Various phases in surface movements linked to deep coal longwall mining: from start-up till the period after closure. International Journal of Coal Science \& Technology 8 (3):412-426. doi:10.1007/s40789-020-00325-0

Wallace L, Lucieer A, Watson CS (2014) Evaluating tree detection and segmentation routines on very high resolution UAV LiDAR ata. IEEE Transactions on
Geoscience and Remote Sensing, vol 52. doi:10.1109/TGRS.2014.2315649

Younger PL, Wolkersdorfer C (2004) Mining impacts on the fresh water environment: technical and managerial guidelines for catchment scale management. Mine water and the environment 23:s2s80

Zhang Z, Li Z, Tian X (2018) Vegetation change detection research of Dunhuang city based on GF-1 data. International Journal of Coal Science \& Technology 5 (1):105-111. doi:10.1007/s40789-0180195-4 